\title{
Stabile SERS Encoded Silver Silica Nanocomposites for Industrial Labeling - The Case of COVID-19 Diagnosis
}

\section{Can Xiao}

University Pompeu Fabra

Bernat Mir Simón

University Pompeu Fabra

Pilar Rivera-Gil ( $\sim$ pilar.rivera@upf.edu )

University Pompeu Fabra

\section{Research Article}

Keywords: SERS-codified plasmonic silica nanocomposites, metallic-thiol bond, controlled agglomeration, COVID-19 SERS-based ELISA assay, industrial labelling/biosensing.

Posted Date: January 3rd, 2022

DOI: https://doi.org/10.21203/rs.3.rs-957554/v2

License: (c) (i) This work is licensed under a Creative Commons Attribution 4.0 International License. Read Full License 
Stabile SERS encoded silver silica nanocomposites for industrial labeling - the case of COVID-19 diagnosis

Can Xiao ${ }^{\mathrm{a}}$, Bernat Mir de Simón ${ }^{\mathrm{a},{ }^{*}}$, Pilar Rivera-Gil ${ }^{\mathrm{a}, *}$

a Integrative Biomedical Materials and Nanomedicine Lab, Department of Experimental and Health Sciences, University Pompeu Fabra, Doctor Aiguader 88, 08003 Barcelona, Spain.

* corresponding authors: bernat.mir@upf.edu ; pilar.rivera@upf.edu

\begin{abstract}
:
Biosensors, especially those with a SERS readout, are required for an early and precise healthcare diagnosis. Unreproducible SERS platforms hamper clinical SERS. Here we report a synthetic procedure to obtain stabile, reproducible and robust highly-SERS performing nanocomposites for labelling. We control the NPs agglomeration and codification which results in an increased number of hot spots, thus exhibiting reproducible and superior Raman enhancement. We studied fundamental aspects affecting the plasmonic thiol bond resulting in $\mathrm{pH}$ exhibiting a determining role. We validated their biosensing performance by designing a SERS-based ELISA SARS-CoV2 detections assay which exhibits limits of detection below $0.01 \mathrm{ng} / \mu \mathrm{L}$.
\end{abstract}

Keywords: SERS-codified plasmonic silica nanocomposites, metallic-thiol bond, controlled agglomeration, COVID-19 SERS-based ELISA assay, industrial labelling/biosensing. 

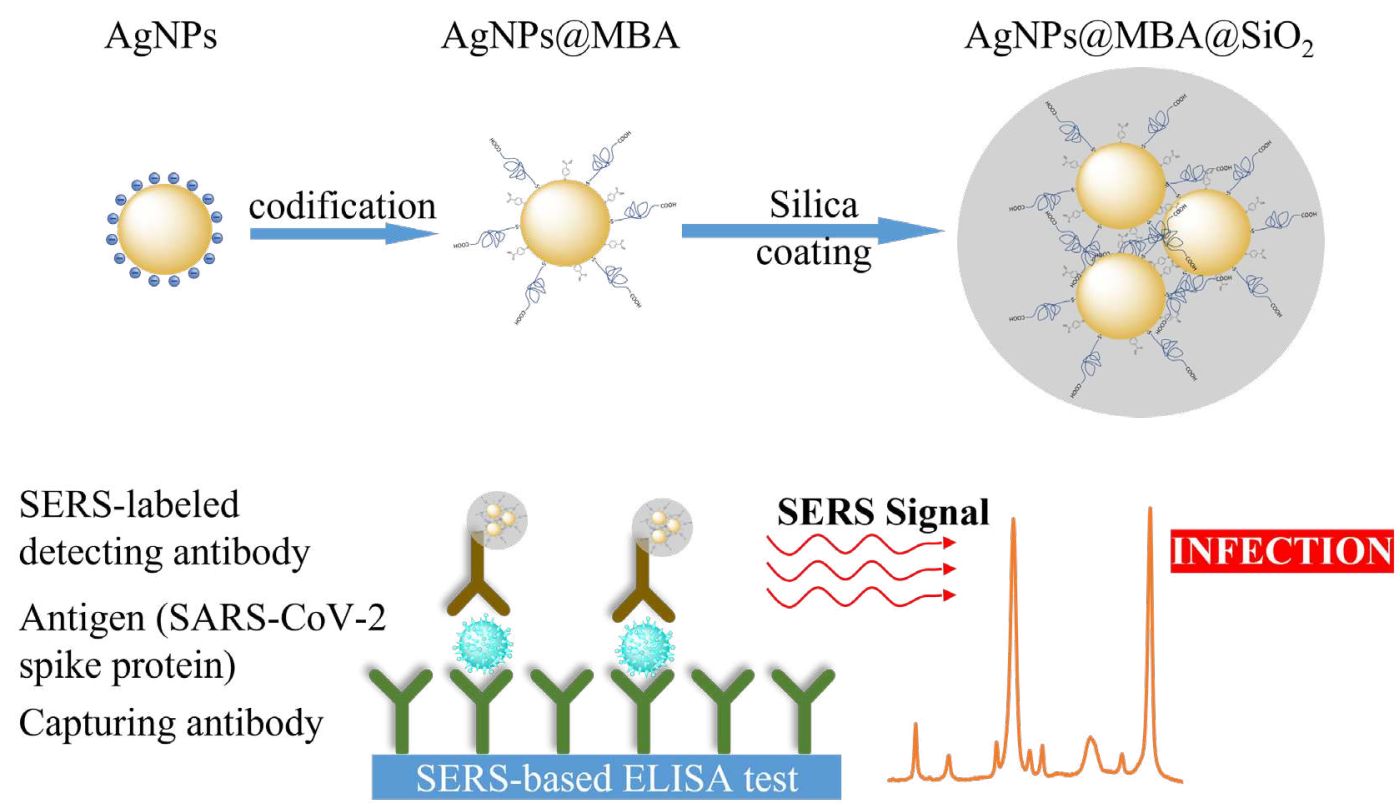

Graphical abstract: Scheme of the rational and controlled synthesis of robust and stabile SERS encoded plasmonic-silica nanocomposites for enhanced, fast, sensitive, and selective labeling and biosensing. 


\section{INTRODUCTION:}

The design for precise diagnosis is critical to human health as for preventing pandemics or other biothreads. Nanosystems has been widely developed in sensor devices for diagnostics, in vitro and in vivo diagnosis(1). This diagnosis field takes benefits from the design and synthesis of nanomaterials, especially noble metal nanomaterials showing localized surface plasmon resonance (LSPR) properties. This LSPR phenomenon limits nanomaterials absorbing specific region of light, and makes the nanomaterials sensitive to the modifications of physical properties of nanomaterials and their environments showing plasmonic absorption shifts(2). Based on their optical properties, flexible functionalized nanomaterials have already been applied in sensing varieties of biomolecules, including biomarkers for cancer(3), enzyme(4), DNA(5), and other biological species(6).

Surface enhanced Raman spectroscopy (SERS) also depends on plasmonic platforms. SERS signals can be collected from the molecules which are in close proximity of nanometallic surfaces with confined LSPR(7). In general, average enhancement factors for typical SERS substrates are amplified by $10^{6}$ to $10^{8}$ orders of magnitude comparing with their Raman signature(8). Moreover, this enhancement can be further increased up to $10^{15}$ orders of magnitude to SERS signal by hot spots(9). Hot spots are highly localized regions of intense local field enhancement caused by the plasmonic coupling of the particles when they are very close to each other(8). Thus, hot spots have critical importance when designing a SERS nanostructure. Gold and silver are the most commonly applied materials for SERS substrates, as they offer high field enhancement in the visible to near infrared range due to their high density of electrons(10). SERS as a non-destructive technology provides chemical information in aqueous environments. Thus SERS has been an effective tool to realize qualitative and quantitative detection of biological species, including micro RNA analysis(11), enzyme(12), hydrogen peroxide(13), staphylococcal enterotoxin $\mathrm{B}(14)$ and other diseases biomarkers (15)'(16) with gold nanowire, gold-silver alloy NPs and gold- $\mathrm{MnO}_{2}$ core-shell, hollow gold nanospheres and gold nanostar and nanosphere separately. However, producing homogenous, sensitive and reproducible SERS platforms is the main difficulty which hampers SERS bioapplications(17), with many efforts made in the design and synthesis of uniform nanomaterials (18),(19),(20). The design and controllable synthesis of nanostructures are critical steps towards implementing SERS in medicine.

Here we present a controllable design and synthesis of a nanostructure confining encoded silver nano-agglomerates inside silica coating (AgNPs@MBA@SiO $\mathrm{SiO}_{2}$. We systematically studied the fundamental aspects and optimized the thiol silver bonding for encoding silver nanoparticles. With the help of controlled agglomeration, we improved the percentage of hot spots which guaranteed the extremely high Raman enhancement. These encoded agglomerates were further encapsulated with a silica shell to protect them from oxidation, contaminations and increase the stability for a long period of time thanks to the unique properties of the $\mathrm{SiO}_{2}$ layer (e.g., surface chemistry, biocompatibility, optical transparency, and colloidal stability). With further biofunctionalization with antibody, we also demonstrated the performance of our devices for SARS-CoV-2 spike protein detection. The reference diagnosis for SARS-CoV-2 is based on reverse transcription polymerase chain reaction $(\mathrm{RT}-\mathrm{PCR})(21)$. Yet there are false positive or 
negative reports, especially for the early stages. Biosensors as alternative or supplementary solutions have been developed based on plasmonic nanomaterials. Gold nanoparticles have been developed for colorimetric detection of SARS-CoV-2 with isolated RNA samples(22) and for IgM Antibodies against the SARS-CoV-2 virus detection based on a lateral flow device(23). A more sophisticated devises based on gold nanoislands was reported for SARS-CoV-2 detection targeting selected sequences(24). Compared with the biosensors mentioned, we selected the detection of SARS-CoV-2 spike protein, which is the major immunodominant protein(25). One of the promising advantages of our biosensor is that it can potentially screen SARS-CoV-2 without prior sample treatment. This whole designed procedure provides our nanostructure high Raman enhancement and robust intensity for sensitive sensing, and uniform synthesis for stability and repeatability. We offered an optimized, stabile nanostructure which can be reliably applied in biosensing.

\section{METHODOLOGY}

A detailed description of the methodology and additional results are presented in the supporting information file.

\section{$\operatorname{AgNPs@MBA@SiO2~Synthesis~}$}

In briefly, we performed a bottom up synthesis of AgNPs relying on the chemical reduction of metal salts and controlling the shape and size by citrate reduction of $\mathrm{AgNO}_{3}$ (26). Then, we co-adsorbed and covalently bound onto the metallic AgNPs surfaces a SERS probe 4-mercaptobenzoic acid (MBA) and a polymeric stabilizer CTPEG12. Finally, we controlled their agglomeration and encapsulation within a homogenous layer of silica following a modification of the Stöber method(27).

\section{SERS based Elisa biosensing:}

AgNPs@MBA@SiO 2 were biofunctionalized with SARS-CoV/SARS-CoV-2 Spike antibody (AgNPs@MBA@SiO2@Ab) via GPTMS. Then SERS based Elisa biosensing followed a sandwich-based diagnostic assays as described in detail in supporting information. The limit of detection (LOD) was calculated by measuring the intensity at $1075 \mathrm{~cm}^{-1}$ with the presence and the absence of antigen, with a 3:1 ratio threshold (Signal / Noise $=3$ ). 


\section{RESULTS AND DISCUSSION}

\section{AgNPs@MBA@SiO2 synthesis and characterization}

We have synthesized a SERS encoded core-shell nanostructure comprising silver NPs agglomerates. Figure 1A shows the geometry of AgNPs@MBA@SiO 2 . The plasmonic encoded nanoagglomerates are within a silica layer. This layer offers unique properties (e.g., surface chemistry, biocompatibility, optical transparency, and colloidal stability). As a result, it protects the nanoagglomerates from oxidation and signal-contamination, thus ensuring a long-term SERS signal stability. The AgNPs were spherical with an anisotropy aspect ratio close to 1. We analysed more than 100 AgNPs@MBA@SiO $\mathrm{SiO}_{2}$ from the TEM images. Around 1\% of the particles were non-spherical, i.e., rod-shaped particles or quasi-flat triangles. More than $60 \%$ of the agglomerates were isolated dimers, trimers, tetramers, pentamers, hexamers, or mixtures thereof. Each NP have four different angles for size measurement. The AgNPs core was approx. 60-70 nm and the $\mathrm{SiO}_{2}$ layer was around $20 \mathrm{~nm}$. The complete size of AgNPs@MBA@ $\mathrm{SiO}_{2}$ was around $110 \mathrm{~nm}$ (figure 1B) and their hydrodynamic diameter 133.8 (PDI 0.130) with a zeta potential of -24.7 ( $c f$., SI, figure SI-1). Further characterization of the NPs was performed with the UV-Vis spectrum(28). Figure 1C shows the normalized extinction spectra of the individual, citrate capped AgNPs and the nanoagglomerates, AgNPs@MBA@ $@ \mathrm{SiO}_{2}$. The characteristic spherical AgNPs' LSPRs is centred around 430 nm. As for AgNPs@MBA@SiO appears a new prominent shoulder absorption at around $700 \mathrm{~nm}$. This red-shift and broadening of the LSPR to higher wavelengths confirms the formation of nanoagglomerates.

Aromatic compounds are commonly used as SERS or Raman probes as they have high Raman cross section. Previous reported articles used ethanol as solvent during Raman probe modification based on the consideration of low solubility of hydrophobic Raman probes(29). However, to the best of our knowledge, there is still lack of detailed information on the fundamental factors affecting Raman probes NPs' modification. Therefore, we studied the formation of thiol-silver bond as a function of $\mathrm{pH}$ and solvent within this work. The SERS probe used here is an aromatic molecule MBA which has high Raman cross section(30) (31) and the polymeric stabilizer CTPEG12 is an aliphatic chain polymer with 12 carbon atoms and a carboxylic group at the end of the chain. Both MBA and CTPEG12 were bonded to AgNPs through their thiol group by forming covalent bonds.

To study the $\mathrm{pH}$ and solvent effect on MBA adsorption, we monitor the evolution of the characteristic ring breathing band of adsorbed MBA at $1075 \mathrm{~cm}^{-1}$ over the time(32) and under different conditions (figure 1D and 1E). AgNPs@MBA were dispersed in 50\% $\mathrm{EtOH} /$ water solution (figure 1D and $c f$., SI, figure SI-2) or in in aqueous solution (figure $1 \mathrm{C}$ and $c f$., SI, figure SI-3) containing no or different amount of $\mathrm{NaOH}$ from 10 min to $24 \mathrm{~h}$. Water and ethanol have different physical property (e.g., pKa and dielectric constant), therefore, it was not surprising to measure different $\mathrm{pH}$ values for each of the solvents even with the same amount of $\mathrm{NaOH}(33)$ (cf., SI, table SI-1). 
Figure 1D and 1E shows the absorption and bonding kinetic results of MBA onto the AgNPs under the different conditions ( $\mathrm{pH}$ and solvent) and over time. When comparing the first 10 min after the addition of Raman code in both solvents, the intensity at 1075 $\mathrm{cm}^{-1}$ increased along with the system $\mathrm{pH}$ and amount of added $\mathrm{NaOH}$. The codification reached a plateau intensity in a $\mathrm{pH}$ dependent manner being shorter under increased alkaline conditions. The adsorption of thiol onto silver mental surface follows physisorption to chemisorption where the $\mathrm{S}-\mathrm{H}$ bond breaks and forms silver thiol covalent bond(34). Environmental $\mathrm{pH}$ will affect the deprotonation of thiol group, thus will affect the formation speed of silver thiol bond. As we observed in our system, an alkaline environment facilitates the codification of MBA onto silver metallic surfaces.

A

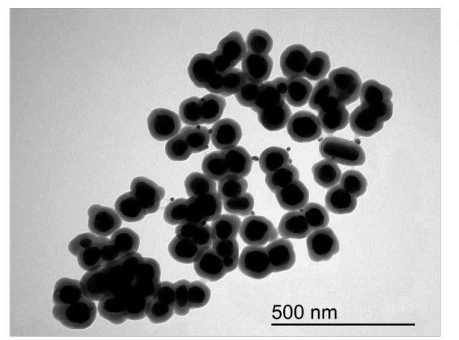

D

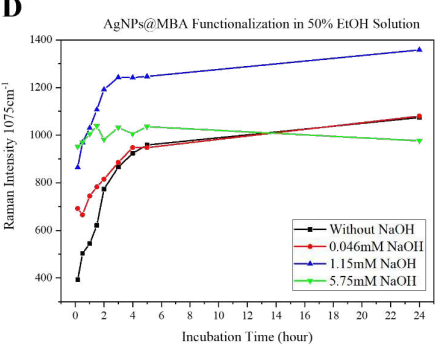

B
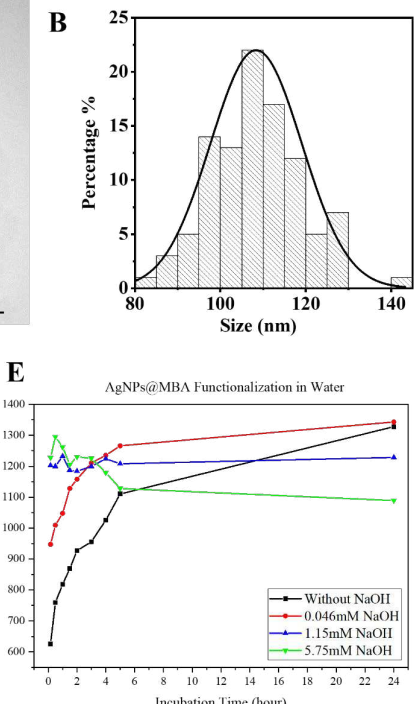

C

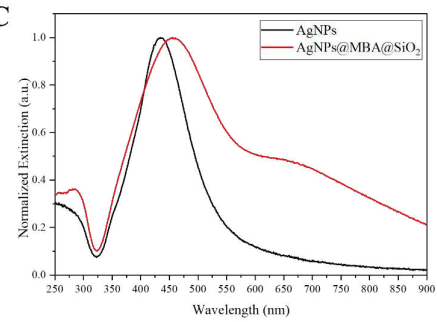

$\mathbf{F}$

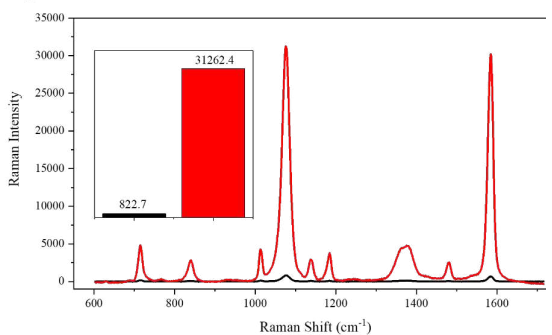

Figure 1: Synthesis and characterization of the SERS-responsive nanoagglomerates. (A) TEM image of AgNPs@MBA@SiO quantification of 100 NPs from TEM images. (C) UV-Visible extinction spectra of AgNPs (black) and AgNPs@MBA@SiO 2 (red). (D and E) MBA adsorption kinetic under different conditions (with or without $\mathrm{NaOH}$ ) measured by SERS intensity at $1075 \mathrm{~cm}^{-1}$ (D) in $50 \% \mathrm{EtOH} /$ water solution and $(\mathbf{E})$ in aqueous solution. Black line: without $\mathrm{NaOH}$; red line: with $0.046 \mathrm{mM} \mathrm{NaOH}$; blue line: with $1.15 \mathrm{mM} \mathrm{NaOH}$; green line: with $5.75 \mathrm{mM} \mathrm{NaOH}$. (F) SERS spectra and SERS intensity at 1075 $\mathrm{cm}^{-1}$ (inset image) of non-agglomerated AgNPs@MBA@ $@ \mathrm{SiO}_{2}$ (in black), and nanoagglomerates,AgNPs@MBA@ $\mathrm{SiO}_{2}$ (in red).

When comparing the plateau intensity after $24 \mathrm{~h}$, the number of adsorbed MBA were similar when the codification was performed in $50 \%$ ethanol/water solution with 1.15 $\mathrm{mM} \mathrm{NaOH}(\mathrm{pH} \mathrm{8)}$ and in aqueous solution with $0.046 \mathrm{mM} \mathrm{NaOH}(\mathrm{pH} \mathrm{8.5).} \mathrm{These}$ conditions exhibited the maximum SERS intensity among all. Subsequent additions of $\mathrm{NaOH}$ and $\mathrm{pH}$ increase resulted in a MBA codification decrease in both solvents. Considering that the pKa of MBA and CTPEG12 is around 4 to 5(35), the deprotonated MBA and CTPEG12 are both negatively charged. This electrostatic repulsion on the surface and the competition between MAB and CTPEG12 will hamper MBA bonding from the bulk solution, since in general, compared with aromatic thiols, aliphatic thiols have better electrochemical and thermodynamic stability(36). Dissociation was also favored at higher $\mathrm{pH}(37)$, which could also be related to this dynamic equilibrium. 
Ethanol has little effect on the modification and stability of metal-thiol bond. Although it was demonstrated that because of the reduction effect of ethanol on gold, the strength of metal-thiol contacts can be weakened(38), ethanol is still recommended for hydrophobic Raman probes.

The number of MBA molecules adsorbed on the metallic surface and the packing of the monolayer is discussed within this work. We show that they depend on factors like the metal-thiol bonding formation speed, which is affected by $\mathrm{pH}$, the electrostatic repulsion on the metallic surface, and the competition between Raman probe and stabilizer. Controlling the $\mathrm{pH}$ in reaction is especially critical to achieve optimized codification of metallic surfaces with thiolated aromatic compounds.

By controlling the agglomeration of AgNPs@MBA and the MBA adsorption, we managed to increase the SERS efficiency up to nearly 40 times when comparing the SERS spectra (characteristic peak intensity at $1075 \mathrm{~cm}^{-1}$ ) of AgNPs@MBA@ $@ \mathrm{SiO}_{2}$ and nonagglomerated AgNPs@MBA@SiO $\mathrm{Si}_{2}$ (figure 1F). This high SERS response was attributed to active SERS structures called "hot spots"(8). In comparison to aggregation or uncontrolled agglomeration, where the NPs merged without hot spots(29), controlled agglomeration offers a useful tool to rationally design nanomaterials for SERS labelling. Our optimized protocol for synthesizing stabile SERS encoded core-shell nanostructure was further confirmed with gold nanoagglomerates ( $c f$., SI, figure SI-5).

\section{Stability of the AgNPs@MBA@SiO2 SERS signal after labelling different types of substrates}

We analysed the SERS signal robustness and stability of our AgNPs@MBA@SiO 2 by depositing them onto 10 different substrates made of different materials (cf., SI, figure SI-6). Figure 2 shows corresponding SERS spectra and zoomed characteristic MBA peak at $1075 \mathrm{~cm}^{-1}$ of AgNPs@MBA@ $\mathrm{SiO}_{2}$ demonstrating efficient labelling of all substrates.
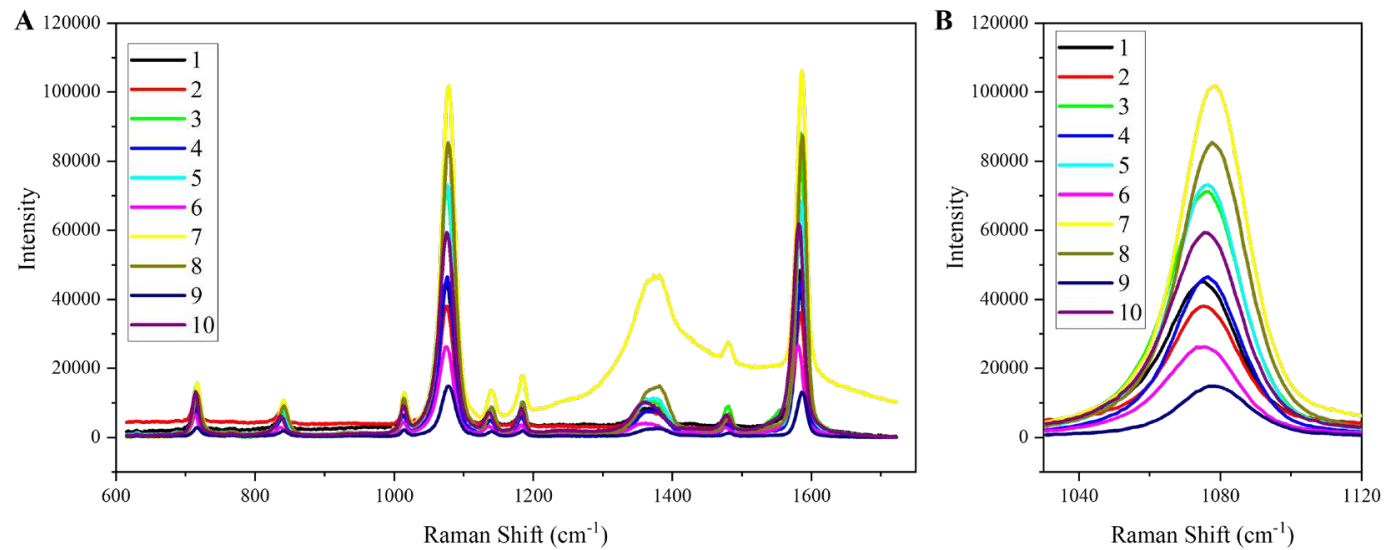

Figure 2: SERS characterization of different substrates wherein the AgNPs@MBA@SiO ${ }_{2}$ has been placed. (A) SERS spectra and (B) zoomed spectra showing characteristic peak at $1075 \mathrm{~cm}^{-1}$ of AgNPs@MBA@SiO 2 on different materials: 1, semianiline leather; 2, aniline leather; 3, pigmented leather; 4, polyester; 5, silk; 6, plastic (PVC); 7 , glass; 8 , brass; 9, cotton; and 10, dyed pigmented leather. 
Except for glass which shows a broad band between 1000 to $1800 \mathrm{~cm}^{-1}$, the background signal originated from all substrates was neglectable. Regardless, both effects did not affect the characteristic bands of MBA at $1075 \mathrm{~cm}^{-1}$ which is used for tracing and labelling. We did observe an uneven SERS intensity between all substrates that can be explained by differences in the distribution of AgNPs@MBA@ $\mathrm{SiO}_{2}$ within the different substrates.

We decided to examine the AgNPs@MBA@ $@ \mathrm{SiO}_{2}$ distribution within the different substrates and its impact on the NPs' geometry (figure 3). For example, in the case of cotton (figure 3B), the NPs are deposited onto the cotton fibres and hidden within the fibers, whereas in the leather (figure 3A), the NPs were more localized in the labelling spot (zoomed SEM areas of figure 3A and 3B). SEM images shows that the distribution can vary with surface properties of the substrates like roughness and porosity. Distribution of the NPs within the substrate can influence the SERS signal. Indeed, we observed that the SERS signal for the dye pigmented leather was higher than for cotton (figure 2). The leather is less rough and porous than cotton, thus hindering the diffusion of the AgNPs@MBA@SiO 2 through the substrate and enhancing the SERS signal. At any rate, both substrates demonstrated preservation of the NPs' geometry, thus SERS signal despite the different distribution. For the elemental analysis (figures 3D and 3E), we took two areas of the labeled spot on the leather, one with visible AgNPs@MBA@ $\mathrm{SiO}_{2}$ (spectrum 1) and another with no visible NPs (spectrum 2). Figures 3D confirms the presence of trace elements from the AgNPs@MBA@ $\mathrm{SiO}_{2}$.
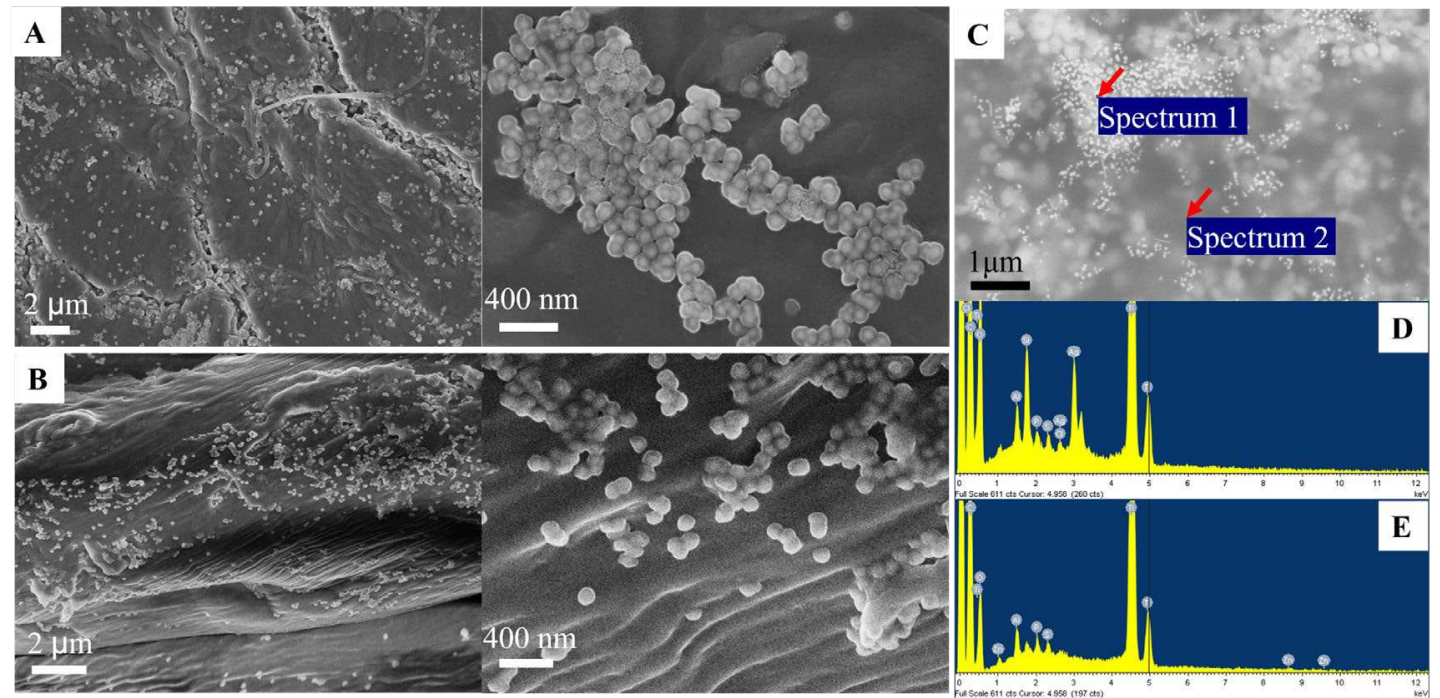

Figure 3: Scanning Electron Microscopy (SEM) and elemental analysis (EDX) of the materials labelled with AgNPs@MBA@SiO . SEM images of leather (A) or cotton (B) labelled with AgNPs@MBA@SiO 2 showing their distribution within the substrate depending on their characteristics (C) SEM images of (A) showing areas with (spectrum 1) and without (spectrum 2) visible NPs that were selected for elemental analysis. (D and E) Their corresponding spectra. The peaks from left to right in (D) correspond to: $\mathrm{Cl}, \mathrm{C}, \mathrm{Ti}, \mathrm{O}, \mathrm{Al}, \mathrm{Si}$, $\mathrm{P}, \mathrm{S}, \mathrm{Ag} / \mathrm{Cl}, \mathrm{Ag}, \mathrm{Ti}, \mathrm{T}$, and in (E) to: C, O, Ti, O, Zn, Al, P, S, Ti, T.

AgNPs@MBA@SiO $\mathrm{SiO}_{2}$ exhibit robust SERS signals; obtained in 0.1 s and with insignificant interfere by other components of the substrate. This provides our SERS- 
responsive NPs with numerous potential applications where high optical signal is needed. The application field ranges from industrial applications where a robust, labeling signal is required or tracing to clinical biosensing and environmental sciences.

Within this work, we demonstrate the capabilities of our AgNPs@MBA@ $\mathrm{SiO}_{2}$ to enhance the sensitivity of ELISA tests for example, to detect immunogenic SARS-CoV2 spike protein, responsible for COVID-19 pandemics.

\section{SERS-based ELISA diagnosis of COVID-19}

Herewith, we prepared our SERS-based ELISA diagnostic kit consisting of a substrate functionalized with the immobilizing antibody, responsible to capture the antigen for further recognition by an anti-antigen antibody providing a readout and the diagnostic result. In our case, the readout is the SERS signal provided by the AgNPs@MBA@ $@ \mathrm{SiO}_{2}$ which recognizes the antigen upon their surface functionalization with the antibody antiSARS-CoV-2-spike protein.

For the surface functionalization of the AgNPs@MBA@ $@ \mathrm{SiO}_{2}$ with the antibody, we first attached a linker (GPTMS) through a reaction called epoxy-silanization(40). It enriches the NPs' surface with epoxy groups which are highly active towards amino groups. Thus, forming covalent bonds between antibodies and GPTMS-NPs. The obtained AgNPs@MBA@SiO $\mathrm{S}_{2} @ \mathrm{Ab}$ recognize the immunogenic SARS-CoV-2-spike protein ( $c f$. , SI, figure SI-7).

Once we engineered the detecting antibody with a SERS readout, we prepared the ELISA plate by coating the substrate with an anti-SARS-CoV-2 spike protein capturing antibody. Upon addition of the spike protein and rinsing the unbounded molecules, we add our AgNPs@MBA@SiO $@$ @Ab. The NPs recognize the antigen protein via a second recognition site and are immobilized onto the ELISA plate substrate. This recognition provides a positive SERS signal (enhancement of the $1075 \mathrm{~cm}^{-1}$ band) thus confirming virus detection (figure 4). The signal was fast (1 s) and specific for the viral protein. Neither the ELISA plate ( $c f$., SI, figure SI-7) nor the absence of antigenic SARS-CoV-2 spike protein (figure 4A) and/or the absence of capturing antibody (figure 4A) provided a SERS positive signal. In the absence of capturing antibody, we increased the concentration of antigen one order of magnitude to detect possible unspecific interactions of the antigen with the plate that could be responsible for false positive results but we did not detect signal. Only when the viral antigen was immobilized, the AgNPs@MBA@SiO $@$ @Ab provided a strong SERS enhancement of the specific band at $1075 \mathrm{~cm}^{-1}$. The enhancement was 20 times higher than in the control samples.

We further conducted SERS-based ELISA tests with a concentration series of the antigen ranging from 0 to $1.6 \mathrm{ng} / \mu \mathrm{L}$ (figure 4B). The intensity ratio between 0.01 and $0 \mathrm{ng} / \mu \mathrm{L}$ at $1075 \mathrm{~cm}^{-1}$ was close to 6, revealing that the LOD of AgNPs@MBA@SiO $@ @$ Ab for antigenic SARS-CoV-2 spike protein detection should be less than $0.01 \mathrm{ng} / \mu \mathrm{L}$.

We want to mention that when the antigen concentration is higher than $0.1 \mathrm{ng} / \mu \mathrm{L}$, the response is less sensitive to small changes in analyst's concentration (figure 4B). However, the specificity is maintained since we obtained positive signals all over the antigen concentration range. A reduction in the sensitivity, can be neglected in the cases where a qualitative response is required, like for example for the fast diagnosis of viral 
infections.

Our SERS encoded nanocomposites,AgNPs@MBA@SiO $@ @$ Ab, offer a robust and stabile labelling platform with high sensitivity and selectivity that can be applied for example in sandwich-based diagnostic assays.

A

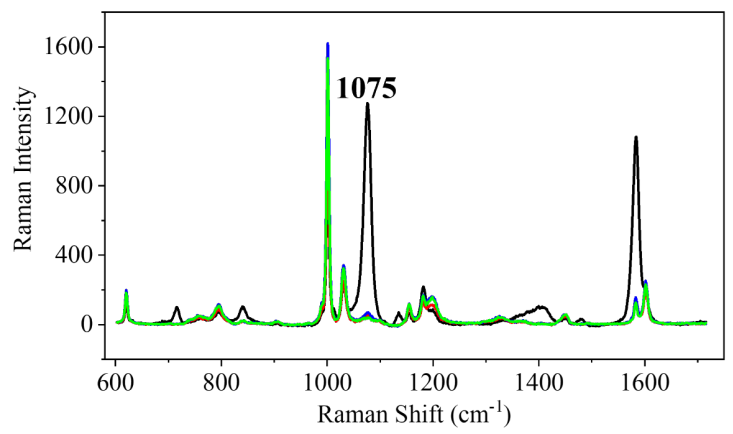

B

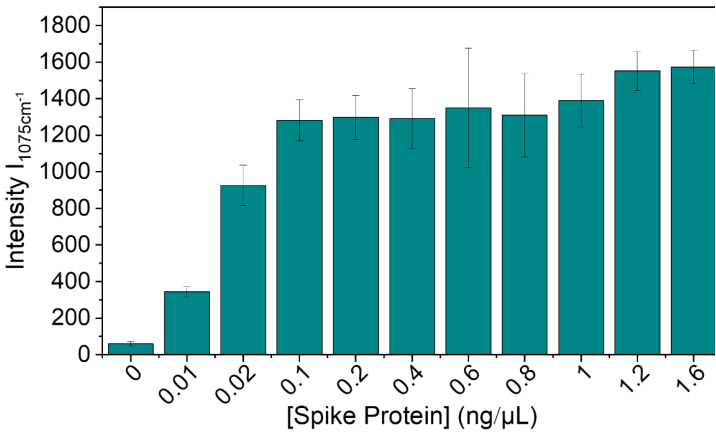

Figure 4: Functional validation of our SERS-based ELISA test. (A) Averaged SERS spectra collected fromAgNPs@MBA@SiO $@$ @Ab in the presence of 0.1 ng/ $\mu \mathrm{L}$ antigenic SARS-CoV2 spike protein (black) and in the absence of antigen (red), capturing antibody (blue) or both (green). In the absence of capturing antibody, the concentration of antigen was increased to 1 ng/ $\mu \mathrm{L}$. (B) SERS intensity at $1075 \mathrm{~cm}^{-1}$ provided by the AgNPs@MBA@SiO $\mathrm{Si}_{2} @ \mathrm{Ab}$ incubated with different amount of antigenic SARS-CoV-2 spike protein (from 0 to $1.6 \mathrm{ng} / \mu \mathrm{L}$ ). The results correspond to an average of 8 spectra recorded from 8 random places in the ELISA plate.

\section{CONCLUSION}

We have rationally designed a stabile synthetic procedure for SERS encoded plasmonicsilica nanocomposites. We have studied fundamental factors affecting the codification of plasmonic NPs with SERS probe for an enhanced labelling efficiency. In general, weak basic environment facilitates the codification of Raman probe onto metallic surface. Continuous increase of the surrounding $\mathrm{pH}$ will favour the dissociation of the metallicthiol covalent bond. Thus, a precise control over the $\mathrm{pH}$ during the codification is critical for an optimized modification of thiolated compound onto metallic surfaces. We also found that ethanol has little effect on the formation of silver thiol bond. The final packing quality of the Raman probe monolayer is affected by the metal-thiol bonding formation speed, the electrostatic repulsion on the metallic surface, and the competition between Raman probe and stabilizer. A precise control over the NPs agglomeration, increases the percentage of hot spots which results in a SERS signal increase of nearly 40 times. This encoded nanoagglomerates were encapsulated and protected by a silica layer which offers the possibility to be multifunctionalized. The nanoaggomerates provide a robust and stable SERS signal with no interference regardless the substrate used. The range of applicability of these nanoagglomerates goes from industrial labeling to environmental science and clinical biosensing, where a fast, in situ, sensitive and specific readout is required. We validated the performance of our nanoagglomerates for the diagnosis of COVID-19. We built up a SERS-based ELISA assay for the fast, sensitive, and specific detection of SARS-CoV-2 antigens. 


\section{ACKNOWLEDGMENTS}

PRG acknowledges the Ministry of Science, Innovation and Universities (MICINN/AEI) (PID2019-106755RB-I00/AEI/10.13039/501100011033, MDM-2014-0370-04, MAT2016-75362-C3-2-R) and the AGAUR (2017 SGR 1054) for financial support.

BM and PRG acknowledge AGAUR (2019 INNOV 00002 [FEDER]).

CX and PRG appreciate the financial support from China Scholarship Council (CSC) (201609110104). 


\section{REFERENCES}

1. Seale-Goldsmith, Mary-Margaret; Leary JF. Nanobiosystems. Wiley Interdiscip Rev Nanomed Nanobiotechnol. 2009;1:553-67.

2. Tiu BDB, Advincula RC. Plasmonics and templated systems for bioapplications. Rend Fis Acc Lincei [Internet]. 2015;26:143-60. Available from: http://dx.doi.org/10.1007/s12210-015-0416-3

3. Im H, Shao H, Park Y Il, Peterson VM, Castro CM, Weissleder R, et al. Labelfree detection and molecular profiling of exosomes with a nano-plasmonic sensor. Nat Biotechnol. 2014;32(5):490-5.

4. Kim E, Baaske MD, Schuldes I, Wilsch PS, Vollmer F. Label-free optical detection of single enzyme-reactant reactions and associated conformational changes. Sci Adv. 2017;3(3):e1603044.

5. Wu X, Xu L, Ma W, Liu L, Kuang H, Kotov NA, et al. Propeller-Like NanorodUpconversion Nanoparticle Assemblies with Intense Chiroptical Activity and Luminescence Enhancement in Aqueous Phase. Adv Mater. 2016;28(28):590715.

6. Beeram SR, Zamborini FP. Selective attachment of antibodies to the edges of gold nanostructures for enhanced localized surface plasmon resonance biosensing. J Am Chem Soc. 2009;131(33):11689-91.

7. Hudson SD, Chumanov G. Bioanalytical applications of SERS (surface-enhanced Raman spectroscopy). Anal Bioanal Chem [Internet]. 2009;394(3):679-86. Available from: http://dx.doi.org/10.1007/s00216-009-2756-2

8. Maher RC. SERS Hot Spots [Internet]. Vol. 9783642206, Raman Spectroscopy for Nanomaterials Characterization. 2012. 1-645 p. Available from: https://doi.org/10.1007/978-3-642-20620-7_10.

9. Anderson DJ, Moskovits M. A SERS-active system based on silver nanoparticles tethered to a deposited silver film. J Phys Chem B. 2006;110(28):13722-7.

10. Tabatabaei M, Sangar A, Kazemi-Zanjani N, Torchio P, Merlen A, LagugnéLabarthet F. Optical properties of silver and gold tetrahedral nanopyramid arrays prepared by nanosphere lithography. J Phys Chem C. 2013;117(28):14778-86.

11. Kang T, Kim H, Lee JM, Lee H, Choi YS, Kang G, et al. Ultra-specific zeptomole MicroRNA detection by plasmonic nanowire interstice sensor with Bitemperature hybridization. Small. 2014;10(20):4200-6.

12. Si Y, Bai Y, Qin X, Li J, Li J, Zhong W, et al. Alkyne-DNA-Functionalized Alloyed $\mathrm{Au} / \mathrm{Ag}$ Nanospheres for Ratiometric Surface-Enhanced Raman Scattering Imaging Assay of Endonuclease Activity in Live Cells. Anal Chem. 2018;90(6):3898-905.

13. Zhang C, Liu X, Xu Z, Liu D. Multichannel Stimulus-Responsive Nanoprobes for $\mathrm{H} 2 \mathrm{O} 2$ Sensing in Diverse Biological Milieus. Anal Chem. 2020;92(18):12639-46.

14. Hwang J, Lee S, Choo J. Application of a SERS-based lateral flow immunoassay strip for the rapid and sensitive detection of staphylococcal enterotoxin B.

Nanoscale. 2016;8(22):11418-25.

15. Villa JEL, Garcia I, Jimenez de Aberasturi D, Pavlov V, Sotomayor MDPT, LizMarzán LM. SERS-based immunoassay for monitoring cortisol-related disorders. Biosens Bioelectron [Internet]. 2020;165:112418. Available from: https://doi.org/10.1016/j.bios.2020.112418 
16. Plou J, García I, Charconnet M, Astobiza I, García-Astrain C, Matricardi C, et al. Multiplex SERS Detection of Metabolic Alterations in Tumor Extracellular Media. Adv Funct Mater. 2020;30(17):1910335.

17. Tripp RA, Dluhy RA, Zhao Y. Novel nanostructures for SERS biosensing. Nano Today. 2008;3(3-4):31-7.

18. Bastús NG, Merkoçi F, Piella J, Puntes V. Synthesis of highly monodisperse citrate-stabilized silver nanoparticles of up to $200 \mathrm{~nm}$ : Kinetic control and catalytic properties. Chem Mater. 2014;26(9):2836-46.

19. Bastús NG, Comenge J, Puntes V. Kinetically controlled seeded growth synthesis of citrate-stabilized gold nanoparticles of up to $200 \mathrm{~nm}$ : Size focusing versus ostwald ripening. Langmuir. 2011;27(17):11098-105.

20. Genç A, Patarroyo J, Sancho-Parramon J, Bastús NG, Puntes V, Arbiol J. Hollow metal nanostructures for enhanced plasmonics: Synthesis, local plasmonic properties and applications. Nanophotonics. 2017;6(1):193-213.

21. Corman V, Landt O, Kaiser M, Molenkamp R, Meijer A, Chu DK, et al. Detection of 2019 -nCoV by RT-PCR. Euro Surveill. 2020;25(3):2000045.

22. Moitra P, Alafeef M, Alafeef M, Alafeef M, Dighe K, Frieman MB, et al. Selective Naked-Eye Detection of SARS-CoV-2 Mediated by N Gene Targeted Antisense Oligonucleotide Capped Plasmonic Nanoparticles. ACS Nano. 2020;14(6):7617-27.

23. Huang C, Wen T, Shi FJ, Zeng XY, Jiao YJ. Rapid Detection of IgM Antibodies against the SARS-CoV-2 Virus via Colloidal Gold Nanoparticle-Based LateralFlow Assay. ACS Omega. 2020;5(21):12550-6.

24. Qiu G, Gai Z, Tao Y, Schmitt J, Kullak-Ublick GA, Wang J. Dual-Functional Plasmonic Photothermal Biosensors for Highly Accurate Severe Acute Respiratory Syndrome Coronavirus 2 Detection. ACS Nano. 2020;14(5):526877.

25. Qiu M, Shi Y, Guo Z, Chen Z, He R, Chen R, et al. Antibody responses to individual proteins of SARS coronavirus and their neutralization activities. Microbes Infect. 2005;7(5-6):882-9.

26. Lee PC, Meisel D. Adsorption and surface-enhanced Raman of dyes on silver and gold sols. J Phys Chem. 1982;86(17):3391-5.

27. Wong YJ, Zhu L, Teo WS, Tan YW, Yang Y, Wang C, et al. Revisiting the Stöber method: Inhomogeneity in silica shells. J Am Chem Soc. 2011;133(30):11422-5.

28. Paramelle D, Sadovoy A, Gorelik S, Free P, Hobley J, Fernig DG. A rapid method to estimate the concentration of citrate capped silver nanoparticles from UV-visible light spectra. Analyst. 2014;139(19):4855-61.

29. Mir-Simon B, Reche-Perez I, Guerrini L, Pazos-Perez N, Alvarez-Puebla RA. Universal one-pot and scalable synthesis of SERS encoded nanoparticles. Chem Mater. 2015;27(3):950-8.

30. Michota A, Bukowska J. Surface-enhanced Raman scattering (SERS) of 4mercaptobenzoic acid on silver and gold substrates. J Raman Spectrosc. 2003;34(1):21-5.

31. García-Algar M, Tsoutsi D, Sanles-Sobrido M, Cabot A, Izquierdo-Roca V, Gil HPR. Subcellular Optical pH Nanoscale Sensor. ChemistrySelect. 2017;2(26):8115-21. 
32. Kneipp J, Kneipp H, Wittig B, Kneipp K. One-and Two-Photon Excited Optical $\mathrm{pH}$ Probing for Cells Using Surface-Enhanced Raman and Hyper-Raman Nanosensors. Nano Lett. 2007;7(9):2819-23.

33. Gelsema WJ, de Ligny CL, Remijnse AG, Blijleven HA. pH - Measurements in alcohol - water mixtures, using aqueous standard buffer solutions for calibration. Recl des Trav Chim des Pays - Bas. 1966;85(7):647 - 60.

34. Tielens F, Santos E. AuS and SH bond formation/breaking during the formation of alkanethiol SAMs on Au(111): A theoretical study. J Phys Chem C. 2010;114(20):9444-52.

35. Hiramatsu H, Osterloh FE. pH-controiled assembly and disassembly of electrostatically linked $\mathrm{CdSe}-\mathrm{SiO} 2$ and $\mathrm{Au}-\mathrm{SiO} 2$ nanoparticle clusters. Langmuir. 2003;19(17):7003-11.

36. Jung J, Kang S, Han YK. Ligand effects on the stability of thiol-stabilized gold nanoclusters: Au25(SR)18-, Au38(SR)24, and Au102(SR)44. Nanoscale. 2012;4(14):4206-10.

37. Bhatt N, Huang PJJ, Dave N, Liu J. Dissociation and degradation of thiolmodified DNA on gold nanoparticles in aqueous and organic solvents. Langmuir. 2011;27(10):6132-7.

38. Xue Y, Li X, Li H, Zhang W. Quantifying thiol-gold interactions towards the efficient strength control. Nat Commun. 2014;5:5348.

39. Turkevich J, Stevenson PC, Hillier J. A study of the nucleation and growth processes in the synthesis of colloidal gold. Vol. 11, Discussions of the Faraday Society. 1951. p. 55-75.

40. Banjanac K, Mihailović M, Prlainović N, Ćorović M, Carević M, Marinković A, et al. Epoxy-silanization - tool for improvement of silica nanoparticles as support for lipase immobilization with respect to esterification activity. J Chem Technol Biotechnol. 2016;91(10):2654-63. 


\section{Supplementary Files}

This is a list of supplementary files associated with this preprint. Click to download.

- NanoLuxeSlv06.pdf 